\title{
LA PROPOSITION CONCESSIVE DANS LA POÉSIE DES TROUBADOURS*
}

\author{
Sybille Häfele ${ }^{* *}$
}

\begin{abstract}
Résumé: Cette étude vise à présenter les possibilités de l'expression concessive dans la poésie des troubadours des' XIIe et XIIIe s. D'une figure rhétorique initiale, la concession devient une notion grammaticale dès le XVIIe. Au XLXe s. les grammairiens la définissent comme une proposition qui, tout en mettant obstacle à une action, ne l'empêche pas d'avoir lieu. Une analyse des chansonniers provençaux rélève des rapports de synonymie syntaxique entre les formes concessives et leurs variantes, ce qui semble confirmer les liens de parenté supposés exister entre les manuscrits.
\end{abstract}

Pourquoi étudier la proposition concessive dans la poésie des troubadours du XIIe et XIIIe s.? À partir de l'article de neuf pages ayant pour titre "Du latin à l'ancien occitan: la proposition concessive" de Henrichsen (1972: 295-304) et du supplément de 19 pages “Der konzessive Gedanke im Provenzalischen" de Miltschinsky-Wien (1917: 165-186), nous avons réalisé une thèse dans l'intention de poursuivre et d'approfondir cette question en présentant les diverses possibilités de l'expression concessive en ancien occitan. Contrairement à toute étude linguistique qui a recours à un corpus diversifié et portant sur plusieurs siècles afin de retracer l'évolution et les changements significatifs de la langue étudiée, cette analyse porte essentiellement sur la poésie des troubadours qui présente la partie la plus importante de la littérature occitane médiévale. Malgré ses contraintes prosodiques, nous avons opté dans la thèse citée pour un corpus homogène et cohérent (2608 chansons des 461 troubadours), tout en sachant qu'une telle approche ne peut fournir de conclusions générales, applicables à l'ensemble des écrits occitans et qu'elle restera nécessairement particulière, inexhaustive et partielle.

\footnotetext{
* Recebido para publicação em agosto de 2006.

“" Doutora Pesquisadora do CNRS e do Centre d'Enseignement et de Recherche d'oc - Université de la Sorbonne, Paris IV.
} 
Le terme concession, traduisant le latin concessio dérivé du verbe concedere, appartient jusqu'au XVIe s. au vocabulaire de la rhétorique. Ce n'est qu'en 1845 que le concept grammatical de la concession est formulé pour la première fois par le grammairien romaniste allemand Édouard Mätzner: "La proposition adverbiale concessive est proche parente de la subordonnée hypothétique: elle est aussi une cause posée comme telle; la différence est que cette cause est en même temps niée dans sa conséquence" (cité par MOREL, 1980: 39). Au cours des siècles, la concession englobe trois dénominations: l'adversation, terme utilisé dès le VIe s. chez Priscien, décrit un rapport d'incompatibilité existant dans un système propositionnel binaire. Les grammairiens du XVIIIe s. accordent à la restriction le caractère de correction; ainsi le deuxième énoncé corrige le premier. La troisième notion, la cause inefficace, a été définie par le grammairien suisse Carl Ayer, en 1876 comme "une relation particulière de cause à conséquence qui s'instaure entre le terme concédé et sa contrepartie" (cité par MOREL, 1980: 122). Dans les ouvrages grammaticaux du XXe s., deux types de définition de la relation concessive apparaissent. L'idée d'opposition est élaborée dès 1938 dans la Syntaxe du français de Georges et Robert Le Bidois, et repris par Ferdinand Brunot dans La pensée et la langue. La relation entre concession et causalité est étudiée par Eugen Lerch dans la Historische französische Syntax et adoptée dans la Syntaxe du français contemporain de Kristian Sandfeld. Actuellement, les recherches se focalisent davantage sur les mécanismes logico-sémantiques apparents dans la relation concessive, comme en témoigne l'analyse de Robert Martin qui conjecture l'existence d'une proposition hypothétique sous-entendue: "L'idée sera que, dans tout énoncé concessif, on perçoit, sousjacente, inscrite dans une image d'univers, une relation hypothétique dont l'antécédent est vrai et dont le conséquent est faux" (MARTIN, 1987: 81).

D'après Banniard (1997: 15), la genèse des langues romanes s'amorce à la fin du Haut-Empire (IIIe s.), s'accèlere à la fin de l'Antiquité tardive (VeVIe s.), pour s'achever pendant le très haut Moyen Âge (VIIe-VIIIe s.) dont la période de transition décisive semble se situer entre 650 et 750 . La réforme carolingienne active de 750 aboutit à une amélioration des textes latins tout en creusant l'écart entre le latin et le latin parlé tardif. Celui-ci entre le Ve et le IXe s. se métamorphose en une nouvelle entité langagière, le roman parlé archaïque (ou protoroman). Le déclin des conjonctions concessives latines 
qui peuvent être ramenées à la langue et à la littérature du latin classique ne surprend pas. À cause de leur difficulté d'emploi et du rôle prédominant des structures paratactiques dans les langues vernaculaires, ces locutions n'ont jamais été employées. Les premiers textes littéraires connus et conservés en occitan datent du XIe s.: la traduction partielle du traité philosophique latin du Boecis peut être située dans la première moitié du XIe s. et La chanson de Sainte Foy, poème de caractère religieux, a été sans doute élaborée entre 1060 et 1080. Dans le Boecis, nous pouvons attester l'emploi de la construction paratactique formée du subjonctif imparfait et d'un renforcement adverbial au vers 92:
Hanc no fo
Om, ta gran vertut agues,
Qui sapiencia
Tot compenre pogues.

En outre, nous retrouvons des structures hypotaxiques comme les extensionnelles non scalaires que que et quora que suivies du subjonctif présent. Prenons par exemple le vers 154-155:

E sanctum Spiritum - qui e bos omes descend:

Que que l corps faza, eu vai l'arma dozen.

En ce qui concerne l'expression concessive dans La chanson de Sainte Foy, nous pouvons remarquer que l'auteur privilégie la subordination exprimée par la conjonction hypothétique si au dépens de la parataxe, dont nous n'avons pu relever qu'une seule occurrence, sous forme de disjonction, au vers 450:

O sia jovens o canuz, si de peccaz es peneduz, sempre 1

Venra gaujz e saluz.

En comparant ces deux premiers témoignages de la langue occitane, une forte influence latine peut être décelée dans le Boecis: les tournures utilisées (extensionnelles non scalaires) sont stylistiquement plus élaborées que dans La chanson de Sainte Foy qui se limite à l'emploi de la conjonction si à valeur hypothético-concessive. Cela serait dû à l'ignorance de son auteur ou 
plus probablement à la non-existence de si tot et si be à cette époque. Ce fait reflète sans doute le stade initial de la formation de ces deux locutions concessives les plus répandues.

Dans la poésie occitane, la concession est exprimée par des structures paratactiques et hypotaxiques. Dès les plus anciens troubadours (Marcabru) jusqu'aux troubadours de la deuxième moitié du XIIIe s. (Cerverí), le verbe esser est employé au subjonctif sous forme de disjonction et avec le renforcement adverbial qui prépare la voie à la formation des locutions conjonctives. La question de non per tan que - adverbe de phrase ou conjonction de subordination - reste délicate. Cependant, après avoir passé en revue les attestations de cette forme dans la poésie, l'existence d'une conjonction de subordination non per tan que, telle qu'elle. est certifiée en moyen français, semble peu probable. L'exemple de Guilhem Ademar 202,2 (VI), v. 21-24, montre la difficulté de cette structure. Kurt Lewent (1957: 29) propose la signification "et cependant" qui s'oppose à la traduction de Kurt Almqvist qui cherche à exprimer davantage l'ironie de ce vers:

\section{Q'ieu non vuoill plus portar lo fais, \\ 22 Fassa $\cdot$ l autrui tirar ! Q'ieu'l lais. \\ 23 Non per tan que pros dompna fo, \\ 24 E si er'ad ops de maiso.}

Étant donné les hésitations de Lewent (1957: 29) devant ces vers, nous proposons avec beaucoup de réserve notre interprétation. Quant à la leçon du vers 24, nous optons plutôt pour l'emploi du futur du verbe esser au lieu de l'imparfait: "E si er ad ops de maiso". Ainsi, nous favorisons davantage l'opposition marquée par le passé exprimé par le parfait (pros dompna $f o$ ) et le futur (er ad ops de maiso) qui introduit une nouvelle idée dans la strophe. La présence du $e$ initial au vers 25 peut avoir deux fonctions: si l'on considère la locution conjonctive non per tan que comme conjonction de subordination "unifiée" - ce qu'il nous est impossible d'attester avec certitude - le $\epsilon$ initial peut, selon Jensen (1994: 316, § 733), introduire une principale quand la subordonnée la précède. D'un autre côté, en s'appuyant sur sa fonction de coordonner deux énoncés, la locution non per tan que serait analysée comme un adverbe de phrase qui porte sur l'énoncé. Avec l'intention de rendre apparente 
l'idée de causalité niée, sous-jacente dans cette locution, nous avançons la traduction suivante: "Pourtant [Ce n'est pas parce qu'elle ne fut pas une dame de valeur que je la quitte] elle fut une dame de valeur [auparavant] et ainsi [dorénavant] elle subviendra à ses besoins."

L'interrogation de l'hypotaxe constitue l'axe principal qui donne les résultats les plus fructueux de cette recherche où nous distinguons les concessives extensionnelles non scalaires et scalaires, terminologie formulée par Bernard Fradin (1977). Les chansons étudiées proposent douze formes différentes d'extensionnelles non scalaires: le relatif à extension indéfinie qui que suivi du subjonctif domine par son nombre les autres extensionnelles, par exemple la pièce anonyme 461,81, v. 5-8 qui indique l'emploi très rare du subjonctif imparfait et de l'indicatif présent après l'extensionnelle non scalaire qui que:

\section{Aissi volgr'eu qe'l cors lai fos, 6 Qi qe sai s'enfezes parliers, \\ 7 Mais n'am un ioi que fos entiers \\ 8 Qui que s'en fai tan enveios.}

Il est intéressant de noter que nous trouvons dans l'énoncé au vers 5, le conditionnel II (volgra) dans la principale et le subjonctif imparfait (fos) placé à la rime. Le même jeu modal existe dans les phrases hypothétiques. Ce phénomène peut probablement s'expliquer par une confusion entre l'adverbe aissi et la conjonction hypothétique si. Quant à qui que suivi du subjonctif imparfait, nous pourrions conclure que cette locution a été considérée comme une deuxième subordonnée et de cette manière le mode de la première a été repris. Par ailleurs, nous observons que la seconde concessive au vers 8 est suivie de l'indicatif dépendant de la principale qui emploie l'indicatif. Ajoutons que l'emploi du subjonctif imparfait dans la relative "que fos entiers" après l'indicatif présent figurant dans la principale est courant en ancien occitan.

Simultanément apparaît dans la poésie occitane cui que, datif de qui que dont l'emploi reste cependant restreint. En revanche, la concessive extensionnelle cals que est le plus fréquemment usitée comme synonyme de qui que. La forme cal que, peu courante, est l'équivalent de que que faisant 
déjà son apparition dans le Boecis.

La locution can que présente des aspects multiples: elle peut devenir l'équivalent d'une conjonction de subordination comme l'indique l'exemple de Sordel 437,28 (XXIII), v. 1-3 (1ère m. du XIIIe s.):

Can qu'ieu chantes d'amor ni d'alegrier

Ni de dompnei, er vei que m'a mestier

Q'ieu chant de gerra, e per gerra m'esgau.

Le subjonctif imparfait après can que se trouve dans la protase qui est antéposée à l'apodose où figure l'indicatif présent. Notons que l'apodose est ouverte par l'adverbe temporel er (era, ara). À notre avis, cette syntaxe est fréquemment employée en ancien occitan dans l'intention de renforcer l'opposition entre le passé et le présent du discours. En outre, la locution can que peut traduire une durée ou des moments précis. Ajoutons l'exemple de Guilhem Peire de Cazals 227,10 (III), v. 29-30 qui soulève des questions:

Per qu'ieu non crey qu'enquer, quan que n'esteya,

No m'autrey tot so qu'er mi desautreya!

François-Just-Marie Raynouard interprète le tour "quan que n'esteya" par "quoi qu'il en soit". En revanche, Emil Levy y accorde plutôt une nuance temporelle qu'il traduit par "aussi longtemps qu'elle en hésite". Afin d'éviter cette problématique, Jean Mouzat simplifie la structure en la traduisant par "malgré tout". En adoptant la valeur temporelle exprimée par Emil Levy, nous proposons la traduction suivante: "Pour cela je ne crois pas, aussi longtemps qu'elle en hésite, qu'elle ne peut m'octroyer tout ce qu'elle me dénie en ce moment."

Le relatif indéfini à valeur temporelle coras que, par exemple, figure déjà dans le Boecis. Com que peut se construire avec le verbe aver ou devenir conjonction de subordination. Quant aux locutions figées qui se sont formées à partir de trois extensionnelles non scalaires (cui que, can que et on que), illustrent un aspect intéressant et difficile de nos recherches. La question des tours can que tir et can que tric, pour laquelle nous n'avons trouvé aucun indice bibliographique, reste ouverte. Avec bien des réserves, nous penchons pour une interprétation temporelle, d'autant plus que cette idée est renforcée 
par le fait que la plupart des attestations montrent l'emploi du futur dans l'apodose antéposée. Le troubadour Peire Rogier 356,3 (VII), v. 8-9 emploié cette locution:

No suy, quar no m'en puesc jauzir.

Tot $m$ 'en jauzirai, quan que tir.

En ce qui concerne les concessives extensionnelles scalaires, leur emploi se limite à la suite prépositionnelle $[\mathrm{per}+\mathrm{SN}+$ proposition relative au subjonctif]. Les moyens d'expression concessives les plus usités dans la poésie sont si tot et si be. Leur existence est certifiée dans la poésie avant 1150 et jusqu'à la dernière moitié du XIIIe s. De même, le si tot occitan est précurseur par rapport au catalan où cette forme n'apparaît qu'au XIIIe s. et à l'italien dont se tutto se trouve exclusivement chez Guittone d'Arezzo, poète italien du XIIIe s. Quant à si be, l'espagnol connaît si bien dès ses premiers témoignages écrits. En italien, se be ne fait son apparition que dans la deuxième moitié du XIIIe s. L'équivalent français si bien se trouve à partir du XVIe s.

L'hypothèse de Henrichsen (1955: 299, note 8) selon laquelle la locution si tot si est "une forme plus développée de si tot" dans la poésie de Perdigon 370,10 (VIII), v. 12-15 est sujette à caution:

Pero no $\cdot \mathrm{m}$ ditz volers,

Sitot $s \cdot \mathrm{m}$ venz folors,

Qu'eu de totz mos dolers

Fass' a mi dons clamors.

Après avoir vérifié sur le CD-ROM les occurrences (sitot si et si tot si) dont le nombre reste cependant très restreint, nous y entrevoyons plutôt un emploi adverbial de si et proposons la traduction suivante: "Mais ma volonté ne me commande pas de me plaindre auprès de ma dame de toutes mes douleurs, même si la folie me vainque ainsi"'.

Comme en italien où un pronom personnel ou une forme verbale conjuguée s'intercale dans la locution si ben, la conjonction si tot est utilisée avant tout sous cette forme dans les poèmes de la première moitié du XIIIes. Tout en conservant sa valeur concessive, si tot peut être séparé par un adverbe 
ou par un pronom personnel. La subordonnée concessive ouverte par si tot et si be dévoile trois principes syntaxiques: elle peut être antéposée, intercalée ou postposée à la principale. L'antéposition de la protase peut s'expliquer par sa proche parenté sémantique avec les propositions hypothétiques où la protase est toujours antéposée. Un cas intéressant de cette construction figure chez Guiraut de Calanson 243,11, v. 12-16:

Si tot al cor m'es trop pezan

Pel belh semblan

Amoros ab que m'a conques, $E$ ma folors

No sai si s'er mals o doussors.

W. Ernst (1930: 355) rejette la proposition d'Alfred Jeanroy (1905) qui interprète si tot comme conjonction concessive, car "der nachfolgende Hauptsatz kann doch wohl nicht mit $e$ eingeleitet werden". Ainsi, il propose la lecture d'une hypothétique et de la locution "tot al cor" (ERNST, 1930: $355)$. Cette hypothèse ne nous convainc pas. Suite à la réflexion d'Alfred Jeanroy (1905), nous renvoyons à Jensen (1994: 316, § 732) qui affirme que "l'apodose peut s'ouvrir fréquemment par $e$, quand la protase la précède". A partir de cette explication, dans le cas présent, nous pouvons avancer qu'il s'agit sans doute d'une antéposition de sitot et de l'introduction de l'apodose par $e$. Nous proposons la traduction suivante: "Bien que je sois trop triste dans mon cœur à cause du beau visage par lequel elle [la dame] m'a conquis, je ne sais pas, si ma folie deviendra douleur ou douceur."

L'intercalation constitue une forme particulière de l'antéposition de la protase. Introduite par un adverbe ou une locution causale de l'apodose, la concessive est intercalée dans cette dernière. En émettant des réserves sur ce classement, à savoir qu'il faudrait plutôt y entrevoir une postposition de l'apodose, nous favorisons cependant une antéposition, car l'argument est prononcé en première position, par exemple chez Gausbert de Puycibot 173,6 (VI), v. 17-21:

Que l'orgoillos, si be 's vens,

N'a blasme per tot saubut,

E l'umils, s'om tot lo te 
Vil e bas sotz se,

N'a dreig sivals conogut.

Il est intéressant de s'interroger sur la syntaxe de cet exemple: si be est antéposé, mais intercalé dans une causale introduite par la conjonction que et sujet. La conjonction si tot, elle-même séparée par un pronom personnel, se trouve intégrée dans une deuxième principale ouverte par le coordonnant $e$ et sujet. Le poète a créé un jeu de symétrie entre ces deux arguments (l'orgoillos, si bell'umils, s'om tot), tout en gardant la même syntaxe des concessives.

Un exemple de si tot en postposition a retenu notre attention chez Bonifacio Calvo 101,11a (XIX), v. 15-21:
15 Bonifaci, fols seria,
16 S'ieu midonz, on es beutatz,
17 Pogues aver a ma guia
18 E tener nud'en mos bratz,
19 S'ieu tot parlar no il podia
$20 \mathrm{E} \cdot 1$ vezer me'n fos vedatz,
21 S'ieu aissi non la penria.

La concessive postposée (v. 19) à la principale (fols seria) et entourée de deux subordonnées hypothétiques (v. 16+21) également postposées n'est pas indiquée dans la traduction de Branciforti (1955:146). Probablement "trompé" par la séparation de si tot et par l'emploi du subjonctif imparfait - le seul exemple ayant pu être relevé dans le dépouillement de si tot l'éditeur l'a interprétée comme une troisième hypothétique. Notons que ces trois protases se réfèrent à la même principale. L'exemple montre deux types d'hypothétiques: Henrichsen (1972) parle du type mixte [ (v. 16) si+ subjonctif imparfait, conditionnel I] et du type pur à l'indicatif [(v. 21) si + conditionnel I, conditionnel I]. En ce qui concerne la concessive, deux modes y figurent: l'emploi de l'imparfait (v. 19) s'explique par un besoin prosodique. Le subjonctif imparfait (v. 20) traduit, sans doute, l'influence de la première hypothétique. Malheureusement, il nous est impossible de comparer ce vers avec d'autres manuscrits, car le texte n'est conservé que dans le manuscrit $\mathbf{a}^{\mathbf{1}}$. Enfin, nous optons pour une traduction à caractère concessif qui, selon nous, devrait s'intégrer plus correctement dans le contexte: "Boniface, je serais fou, 
si je pouvais posséder à ma guise ma dame, qui incarne la beauté, et la tenir nue dans mes bras, même si je ne pouvais lui parler et que la regarder me fût interdit, si je ne la prenais pas à cette condition."

L'œuvre de Bertolome Zorzi, troubadour vénitien de la deuxième moitié du XIIIe s., présente d'autres particularités grammaticales. Il est le seul poète à utiliser la forme ben que afin d'exprimer l'idée concessive, par exemple dans la chanson 74,2 (III), v. 72-75:

Pois son pretz a tant cregut,

C'om non a ren tan prezan

Que mais non l'auza vertatz,

Ben c'om l'aja aut estendut

Sobre cels qu'al som estan.

Pourtant, à notre avis, il ne s'agit pas d'une "création" occitane, mais plutôt d'une influence de la locution conjonctive italienne benchè qui voit le jour à la même époque en Italie. De mêne, la forme ja que représente dans cette poésie un emploi isolé. Notons que ja que ne figure en catalan qu'au $\mathrm{XVe}$ s. où il a une fonction causale.

Afin de mettre en lumière les liens de parenté supposés exister entre les manuscrits provençaux, nous avons consacré une étude comparative aux locutions concessives relevées et à leurs variantes respectives - ce qui nous a conduite à observer des rapports de synonymie entre si tot et si be, entre les concessives extensionnelles et parmi les propositions hypothétiques restrictives. La proposition concessive montre également une corrélation syntaxique avec les causales, les hypothétiques et les relatives dans de nombreux chansonniers. Finalement, nous avons pu déceler des groupements de manuscrits qui confirment la tradition manuscrite: 1. Les manuscrits $\mathbf{A B}$ $\mathrm{du}$ XIIIe s. sont, la plupart du temps, très proches. Quand les deux manuscrits adoptent le vers en question, ils coïncident. Le manuscrit $\mathbf{D}$ du XIIIe s., faisant également partie de la tradition vénitienne $(=\varepsilon)$, apparaît à plusieurs reprises à côté de A pour adopter la même variante:

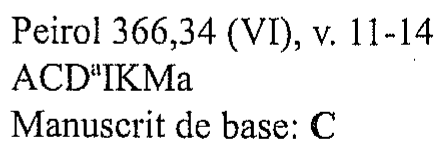


Mout hi trobei amoros' acuynhdansa

$E$ bels solatz e bons ensenhamens;

E si tot sui per s'amistat jauzens, Quar m'es tan lonh, n'ai dolor e pesansa.

Si tot] si ben $\mathbf{A D}^{\prime \prime} \mathbf{I K}$

La constellation jumelle IK et le groupe $\mathbf{A D a}(\mathbf{D a}$ est un appendice de D et fait à partir du Liber Alberici $=\beta$ ) pourraient être ramenés à une source commune $\varepsilon$.

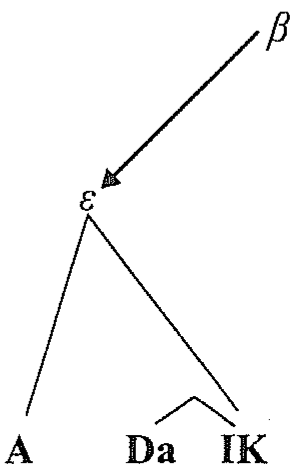

2. Le caractère quasi identique de $\mathbf{I K}$, deux manuscrits italiens du XIVe s., a pu être noté. S'ajoutent à ce groupe, les manuscrits $\mathbf{N}$ (Italie, XIIIe s. (Avalle) ou XIVe s. (Riquer) et $\mathbf{N}^{2}$ (Italie, XVIe s.). La concordance $\mathbb{I K}+\mathbf{d}$ (supplément de $\mathbf{D}$ et copie du XVIe s. de $\mathbf{K}$ ) a pu également être observée. 3. La constellation jumelle de $\mathbf{C R}$, manuscrits languedociens du XIVe s., est moins stable que celle de $\mathbf{A B}$ et de $\mathbb{I K}$, mais apparente:

Peire Cardenal 335,10 (XLV), v. 7-11

TMM ${ }^{2}$ IKdRC

Manuscrit de base: $\mathbf{C}$

Pueis quals que l'aprenda,

Abanz que 1 reprenda,

Regart la razon 
Pueis lo don $0 \cdot 1$ venda

A tal que 1 revenda.

(...)

Quals que] qui que IKdRC

Étant donné l'absence des manuscrits $\mathbf{A}$ et $\mathbf{D}$, représentants les plus typiqués de la famille vénitienne $\varepsilon$, nous pouvons émettre l'hypothèse de l'existence du codice antico qui se divise en deux codices interpositi $\alpha$ et $\beta$ (Liber domini Alberici). Les manuscrits $\mathbf{C R}$ sont à la base de $\boldsymbol{\alpha}$, alors que $\mathbf{I K d}$ peuvent être ramenés à $\boldsymbol{\beta}$.

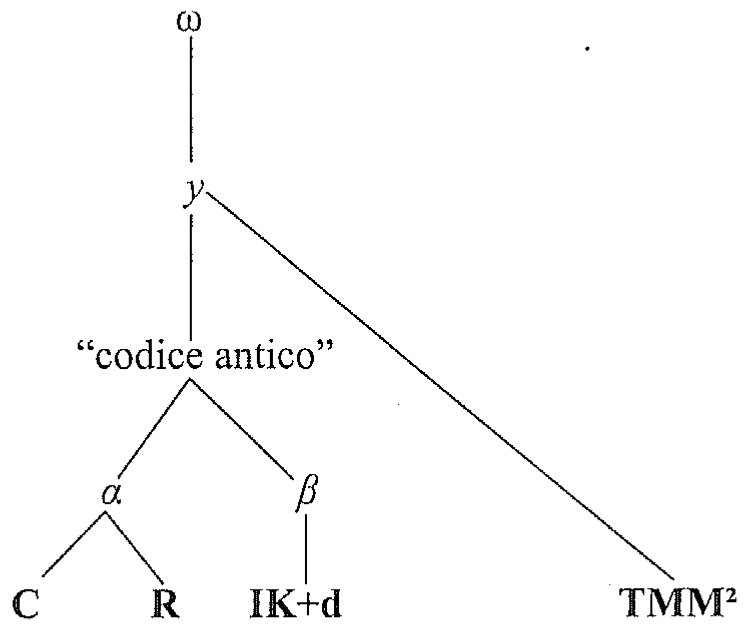

4. Le manuscrit toscan $\mathbf{P}$ daté de 1310 et le chansonnier italien $\mathbf{S}$ du XIVe s. apparaissent parfois ensemble dans les variantes. De plus, le groupe PS-L est présent, en tenant compte du fait que le manuscrit $\mathbf{S}$ a été utilisé pour corriger $\mathbf{L}$, manuscrit italien du XIIIe s.:

Arnaut Daniel 29,6 (II), v. 37-41

ABSPLQGcIKN²DNHECRSg

Manuscrits de base: $\mathbf{A B}$

Si ben vauc per tot ab esdalh,

Mos pessamens lai vos assalh, 
Qu'ieu chan e valh

Pel joi que'ns fim

Lai on partim.

Si ben] si tot Sg, si tot vals (val P) per dos madaill (medaill) LPS

Sg, $\mathbb{L}$ et PS se réfèrent à un subarchétype commun $\alpha$ et se distinguent des autres manuscrits en présentant la conjonction si tot à la place de si be. $\mathbb{L}$ s'attache à PS probablement à la suite de l'utilisation de $\mathbf{S}$ par le copiste afin de corriger le manuscrit $\mathbf{L}$.

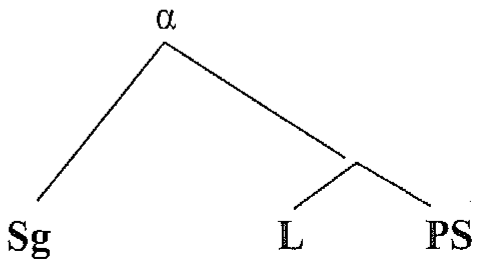

5. Le manuscrit $\mathbf{M}$ copié en Italie au XIVe s. révèle une certaine prédilection pour l'emploi de si tot. Il est évident que $\mathbf{M}$ ne remplace jamais si tot par si be. 6. Le manuscrit catalan $\mathrm{V}$ daté de 1268 propose à plusieurs reprises sa propre interprétation. 7. La copie a du Chansonnier perdu de Bernart Amoros indique de nombreux unica dans les variantes:

Giraut de Borneil 242,19 (XLI), v. 25-27

\section{CDIKRSga}

Manuscrit de base: a

Non pot esser, qe ben o sen, Con qe m'en fenha, ni s'eschai! Ia ses ioi be non chantarai.

\section{Con que] anz que CDIKRSg}

Nous observons que le manuscrit a est le seul à employer la variante con que, tandis que tous les autres manuscrits utilisent la conjonction temporelle anz que. Par conséquent, il est vraisemblable qu'à côté de $y$, a ait puisé dans 
cette source qui lui est spécifique et qui est responsable de ses nombreux unica. D'après D'Arco S. Avalle (1961), des fragments et des vestiges d'autres traditions manuscrites indépendantes sont encore répérables dans le chansonnier de Bernart Amoros, ou par l'intermédiaire de sa copie du XVIe s. $(\mathrm{a}+\mathrm{ca}+\mathrm{Fa})$.

La question ultime qui nous intéresse est de savoir pourquoi les troubadours utilisent fréquemment cette expression. Une explication se trouverait éventuellement dans la fin'amor qui «se veut sublimation du désir, dans l'inachèvement de la conquête.» Ainsi, la concession est un excellent instrument pour mettre en opposition deux idées a priori incompatibles (la dame et le troubadour) et exprimer le désespoir du poète face à ce désir inassouvi.

Resumo: Este estudo visa apresentar as possibilidades da expressão concessiva na poesia dos trovadores dos séculos XII e XIII. De uma figura retórica inicial, a concessão tornase uma noção gramatical, a partir do século XVII. No século $X I X$, os gramáticos a definem como uma proposição que, mesmo colocando obstáculo a uma ação, não a impede de ocorrer. Uma análise dos cancioneiros provençais mostra relações de sinonimia sintática entre as formas concessivas e suas variantes, o que pode confirmar os laços de parentesco, que devem, supostamente, existir entre os manuscritos.

\section{RÉFÉRENCES BIBLIOGRAPHIQUES}

AVALLE, D'A. S. La letteratura medievale in lingua d'oc nella sua tradizione manoscritta. Turino: Giuglio Einaudi Editore, 1961.

BANNIARD, M. Du latin aux langues romanes. Paris: Éditions Nathan, 1997.

BRUNEL-LOBRICHON, G.; Duhamel-Amado, C. Au temps des troubadours: XII'-XIII" siècles. Paris: Hachette Littératures, 1997.

FRADIN, B. Les concessives extensionnelles en français moderne. Thèse de troisième cycle. Université de Paris VIII, 1977.

GRÖBER, G. Die Liedersammlungen der Troubadours. Romanische Studien, n. 2, 1877, p. 337-670. 
HENRICHSEN, A.-J. Les phrases hypothétiques en ancien occitan; étude syntaxique. Bergen: A/S John Griegs Boktrykkeri, 1955.

HENRICHSEN, A.-J. Du latin à l'ancien occitan: la proposition concessive. Mélanges de philologie romane dédiés à la mémoire de Jean Boutière. Soledi: Liège, 1972. I, p. 295-304.

JENSEN, F. Syntaxe de l'ancien occitan. Tübingen: Max Niemeyer Verlag, 1994.

LERCH, E. Historische französische Syntax. Leipzig: O. R. Reisland, 19241934. t. II.

LEVY, E. Provenzalisches Supplement Wörterbuch. 8 t. Leipzig: O. R. Reisland, 1884-1924.

LEWENT, K. Old Provençal Non per tan que, *Non per can que, Non per so que, Non que. The Romantic Review, XLVIII, 1957, p. 28-35.

MARTIN, R. Langage et croyance: les univers de croyance dans la théorie sémantique. Bruxelles: Pierre Mardoya, 1987.

MENARD, P. Manuel du français du moyen âge. 1. Syntaxe de l'ancien français. Bordeaux: Sobodi, 1976.

MILTSCHINSKY-WIEN, M. Der Ausdruck des konzessiven Gedankens in den altnorditalienischen Mundarten nebst einem Anhang das Provenzalische betreffend. Halle (Saale): Max Niemeyer Verlag, 1917. p. 165-186.

MOREL, M.-A. Etude sur les moyens grammaticaux et lexicaux propres à exprimer une concession en français contemporain. Thèse d'État, Lille, 1980.

PULSONI, C. Nell'atelier del correttore del ms. Provenzale L (Vat. Lat. 3206). Actes du I $V^{\circ}$ Congrès International de l'AIEO Vitoria/Gasteiz, 22-28 août 1993, t. I, p. 287-295.

SOUTET, O. Études d'ancien et de moyen français. Paris: PUF, 1992. 


\section{ÉDITIONS}

ALMQVIST, K. (Éd.). Poésies du troubadour Guilhem Adémar. Uppsala: Almqvist \& Wiksells, 1951.

ASTON, S. C. (Éd.). Peirol, Troubadour of Auvergne. Cambridge: Cambridge University Press, 1953.

BRANCIFORTI, F. (Éd.). Le rime di Bonifacio Calvo. Catania: Università di Catania, 1955.

CHAYTOR, H. J. (Éd.). Les chansons de Perdigon. Paris: C.F.M.A., 1926.

ERNST, W. (Éd.). Die Lieder des provenzalischen Trobadors Guiraut von Calanson. Romanische Forschungen, n. 44, 1930, p. 255-406.

EUSEBI, M. (Éd.). Arnaut Daniel, il sirventese e le canzoni, Milano: All'insegna del pesce d'oro, 1984.

HOEPPFNER, E.; ALFARIC, P. (Éd.). La chanson de Sainte Foy. Paris: Société d'Édition Les Belles Lettres, 1926. T. 2.

JEANROY A. (Éd.). Poésies provençales inédites d'après les manuscrits de Paris. Annales du Midi 17, 1905, p.457-468.

LAVAUD, R.; MACHIOT, G. (Éd.). Boecis, poème sur Boèce (fragment), le plus ancien texte litteraire occitan, réédité, traduit et commenté. Toulouse: Institut d'Études Occitanes, 1950.

MOUZAT, J. (Éd.). Guilhem Peire de Cazals, troubadour du XIII siècle Paris: Les Belles Lettres, 1954.

RICKETTS, P. T. (Éd.). COM 1 (Concordance de l'occitan médiéval) Turnhout: Brepols Publishers, 2001.

RIEGER, A. (Éd.). Trobairitz. Tübingen: Max Niemeyer Verlag, 1991. 